\title{
Editorial policies at SWSC
}

\author{
Anna Belehaki ${ }^{1, *}$ and Jean Lilensten ${ }^{2}$ \\ 1 National Observatory of Athens, Institute of Astronomy, Astrophysics, Space Applications and Remote Sensing, \\ 15236 Palaia Penteli, Greece \\ *Corresponding author: e-mail: belehaki@noa.gr \\ 2 Institut de Planétologie et d'Astrophysique de Grenoble, 38041 Grenoble, France
}

Received 8 March 2013 / Accepted 8 March 2013

\begin{abstract}
Reaching the end of the second year of the Journal of Space Weather and Space Climate, we present an overview of important issues that resulted in the clarification of our editorial policy, mainly triggered by the response of the scientific communities related to SWSC. We conclude this editorial with future perspectives concerning the impact factor and the charging policy.
\end{abstract}

Key words. space weather - space climate - strategy - policy sciences - knowledge bases

\section{Introduction}

During the first 14 months of SWSC publications, from August 2011 to October 2012, the journal web site had about 7500 total visits, over 20000 page views and readers from 70 countries around the world who downloaded about 3000 papers. Especially in 2012 we had an average of 1600 abstracts downloaded per month and this figure increased within the first months of 2013. This is an encouraging statistics for a newly born journal. To further strengthen the impact of SWSC, there is continuing interaction between the Editors and the related communities that helped us to better define and refine the editorial policy and to make plans for the future which we report in this editorial.

\section{Editorial policy}

\subsection{Considering a submission for publication}

When a paper is submitted, three people in the Editorial Board first read it. If all three agree to reject it, a report is sent to the authors to explain the reasons of the decision. If at least one amongst the three recommends to proceed further, the paper is assigned to an Associate Editor (AE). The AE may still recommend to reject it, or decide to proceed with the review process. This is a very efficient way, but also very selective. Indeed, even though SWSC accepts manuscripts related to space weather and to space climate from a broad range of fields, we want to set up a high-quality journal: All manuscripts which pass the pre-screening process are peer reviewed according to the quality standards of international scientific journals. These standards are analysed below for each submission type, and relevant criteria are strictly applied. Within the first months after the set up of the journal, to our surprise, we have received articles that could not fit the concept of a peer-review scientific journal. For instance, we received meeting minutes and meeting reports that are not within the scope of our journal. Minutes are very useful for participants of a meeting but of little interest to outsiders. Good places to distribute workshop reports are electronic newsletters which are indeed often used for this purpose. We also received some submissions made from a copypaste of projects submitted to funding agencies. Project proposals or descriptions of intentions are not results and not within the scope of SWSC.

These issues triggered some important discussions within the Editorial Board members that led to the more precise description of the aims and scope of the journal. According to these discussions, we decided to restrict the submissions in three categories:

\subsubsection{Scientific papers}

In this category we welcome manuscripts related to space weather and space climate from a broad range of fields including solar physics, space plasma physics, aeronomy, planetology, radio science, geophysics, biology, medicine, astronautic, aeronautic and electrical engineering, meteorology, climatology, mathematics, economy and informatics. Scientific papers must present new results or ideas as concisely as possible. Manuscripts that are obviously flawed or are of insufficient scientific content are not considered for publication.

\subsubsection{Technical papers}

Manuscripts about new instrumental developments and/or observational procedures are very suitable for publication under the category of technical papers. However, we encourage and expect that such papers will use physical data to demonstrate expected performance or to validate actual performances of these new developments or procedures.

\subsubsection{Papers on education and outreach}

In this category our purpose is to publish the results of research in education, to share experiences and innovations, to learn how to apply education research in the classroom and to communicate the results of interesting projects. Publications on Education and Outreach have as a scope to provide a forum where 
it is possible to learn from others' experience and to disseminate new ideas that can be used both in the classroom and beyond.

The submissions could be of three types: regular papers, invited papers and letters.

Regular and invited papers have practically no limitations in length; however, they must be concise enough. Invited papers are solicited by the EiC or the AEs, and they also follow the standard editorial procedure, as regular papers. Letters must be timely, very targeted on presenting specific findings/discoveries and should not exceed four journal papers.

\subsection{Quality standards}

Quality standards for scientific and technical papers require that all manuscripts submitted for publication in SWSC contain enough new insight; present the results against a properly referenced background of existing work; present adequate evidence that supports the conclusions. For submissions on education and outreach it is required that the papers demonstrate the usefulness of presented ideas, presented in a clear and effective way, and provide enough evidence concerning the validation of the techniques applied.

\subsection{Overview of 2011 and 2012 regular and special issues}

In 2011, 36 papers were submitted. About half of them were rejected directly without going to referees. In 2012 the rejection rate of submitted articles at the pre-screening phase decreased significantly to $19 \%$, but the number of submissions also doubled. This means that the communities related to SWSC started to feel more confident with the journal and submitted for publication their best work.

Another proof of the endorsement of the communities involved is the number of special issues within the first 2 years of the journal life time.

The first special issue on Space Climate had 18 submissions and now it is closed with the publication of 12 papers discussing a broad range of aspects of Space Climate, i.e., the longterm change in the Sun and its effects in the heliosphere (solar wind, HMF, cosmic rays, etc.) and the near-Earth space environment, including magnetosphere, ionosphere, and the solar effects on the atmosphere and climate. This Special issue originates from, but is not restricted to contributions presented at the Space Climate Symposium-4 in January, 2011 in Goa, India, and in the Space Climate Session at the ESWW8 meeting in November 2011 in Namur, Belgium. Chief Guest Editor was Kalevi Mursula, from the University of Oulu in Finland.

The second special issue welcomes contributions on "First results from the EU-FP7 projects on Security of Space Assets from Space Weather Events". The special issue was announced in November 2011. SWSC has received a limited number of submissions maybe because the related FP7 projects were not yet so mature to report specific results. In addition, a large percentage of submitted manuscripts were unfortunately rejected because the contents were mostly an extract of research proposals rather than presentation of research results.

The third special issue is a collection of the final results from the COST Action ES0803 "Developing space weather products and services in Europe". The articles are related to the objectives of COST Action ES0803 as stated in its Memorandum of Understanding. Fourteen manuscripts were submitted, seven are published and few are still under evaluation.
A fourth special issue planned for 2013 invites papers presented in the conference "Space Weather and Challenges for Modern Society" held in Oslo, on 22-24 October 2012. The conference was organized by The International Emergency Management Society (TIEMS) in cooperation with the Norwegian Space Centre, Norwegian Water Resources and Energy Directorate, Norwegian Statnett, Finnish Meteorological Institute, Danish Meteorological Institute, and Swedish Institute of Space Physics, see also http://tiems.info/About-TIEMS/oslo-conference-2012.html. Guest EiC for this special issue is Peter Wintoft from the Swedish Institute of Space Physics.

Special issues are challenging tasks for a variety of reasons. Very often the whole community is involved and it might be difficult to find truly independent and competent reviewers. In other cases the AE must clearly explain to the reviewers the specificities of the special issue that have to be taken into consideration in their evaluation and recommendations. However, we have decided to encourage Special Issues at SWSC because it is an effective way to highlight new results on a specific topic or to present current trends at specific research areas of space weather and space climate.

\section{Outlook}

\subsection{Impact factor}

High quality is the primary scope of SWSC. This is the only way to meet the standards of our community, but also is the means to get high impact factor. The first impact factor for SWSC will be announced in 2014 and until then we are doing our best to advertise the journal as much as possible, increasing the number of submissions without making compromises with the quality standards. Our editors are very supportive and participate actively in this procedure. The annual regular meetings of the Editorial Board and the frequent teleconferences between the EB members help to resolve urgent issues and discuss special cases concerning mainly conflicting views between the editors, the reviewers and the authors. Finally, we point out again that our publisher, EDP Sciences, maintains an electronic open access archive of all published material in SWSC that is indexed in Crossref and therefore in all the international Digital Library reference systems.

\subsection{Publication charges and open access policy}

SWSC is an electronic open access journal. This means that it is totally free for the readers but the drawback is that we have to secure funding for the publication of articles from other sources. So far SWSC was supported with funding from the COST Office through the Action ES0803, and from our publisher EDP Sciences. Significant support is also provided by the STCE of ROB which operates with its own funding the Editorial Office of the Journal. This arrangement allows the journal to operate, for this first period, without charging the authors with publication fees. Within 2013 or in 2014 at the latest, this policy has to change and some charges have to be applied in order to maintain the open access policy, which is also a recommendation of the EU for the publication of results from FP7 projects and from the future Horizon 2020 projects. Until we will have our first impact factor from Thomson, we will try to keep the publication charges at the lowest possible rate, to encourage submissions. 
A. Belehaki and J. Lilensten: Editorial policies at SWSC

The active involvement of our Associate Editors and our publisher helped us tremendously to better specify the editorial policy. But the future of SWSC will be driven by the requirements of the broader scientific communities related to the journal, and it is our primary aim to meet these requirements and to make SWSC a professional journal for validating, communicating and archiving exciting research results.
Acknowledgements. We thank our Associate Editors and our publisher for their helpful comments and advice. We also thank Jan Janssens from STCE for the daily operation of the Editorial Office.

Cite this article as: Belehaki A \& Lilensten J: Editorial policies at SWSC. J. Space Weather Space Clim., 2013,3 , A13. 